This information is current as of April 26, 2023.

\title{
Characteristic MR Imaging Findings of the Neonatal Brain in RASopathies
}

M.N. Cizmeci, M. Lequin, K.D. Lichtenbelt, D. Chitayat, P. Kannu, A.G. James, F. Groenendaal, E. Chakkarapani, S. Blaser and L.S. de Vries

AJNR Am J Neuroradiol 2018, 39 (6) 1146-1152 doi: https://doi.org/10.3174/ajnr.A5611 http://www.ajnr.org/content/39/6/1146 


\title{
Characteristic MR Imaging Findings of the Neonatal Brain in RASopathies
}

\author{
(D) M.N. Cizmeci, (D) M. Lequin, (D).D. Lichtenbelt, (DD. Chitayat, (DP. Kannu, (D)A.G. James, (D) F. Groenendaal, (D)E. Chakkarapani,
} (D) S. Blaser, and IDL.S. de Vries

\begin{abstract}
BACKGROUND AND PURPOSE: Neuroimaging features in neonates with RASopathies are rarely reported, and to date, there are no neuroimaging studies conducted in this population. Our aim was to investigate the occurrence of supratentorial and posterior fossa abnormalities on brain MRIs of neonates with a RASopathy.
\end{abstract}

MATERIALS AND METHODS: An observational case-control study of neonates with a confirmed RASopathy was conducted. The presence of an intraventricular and/or parenchymal hemorrhage and punctate white matter lesions and assessments of the splenium of the corpus callosum, gyrification of the cortical gray matter, and enlargement of the extracerebral space were noted. The vermis height, transverse cerebellar diameter, cranial base angle, tentorial angle, and infratentorial angle were measured.

RESULTS: We reviewed 48 brain MR studies performed at 3 academic centers in 3 countries between 2009 and 2017. Sixteen of these infants had a genetically confirmed RASopathy (group 1), and 32 healthy infants were enrolled as the control group (group 2). An increased rate of white matter lesions, extracerebral space enlargement, simplification of the cortical gyrification, and white matter abnormalities were seen in group $1(P<.001$, for each). The vermis height of patients was significantly lower, and tentorial and infratentorial angles were significantly higher in group $1(P=.01, P<.001$, and $P=.001$, respectively).

CONCLUSIONS: Neonates with a RASopathy had characteristic structural and acquired abnormalities in the cortical gray matter, white matter, corpus callosum, cerebellum, and posterior fossa. This study provides novel neuroimaging findings on supratentorial and posterior fossa abnormalities in neonates with a RASopathy.

ABBREVIATIONS: $\mathrm{NICU}=$ neonatal intensive care unit; NS = Noonan syndrome; $\mathrm{TA}=$ tentorial angle

$\mathbf{R}^{\wedge}$ ASopathies are a family of multisystemic disorders with overlapping phenotypic features affecting approximately 1 in 1000 neonates. ${ }^{1}$ These disorders are caused by germline mutations in genes coding for proteins that are part of the RAS/mito-

Received October 30, 2017; accepted after revision February 5, 2018.

From the Departments of Neonatology (M.N.C., F.G., L.S.d.V.) and Pediatric Radiology (M.L.), Wilhelmina Children's Hospital, University Medical Center Utrecht, Utrecht, the Netherlands; Brain Center Rudolf Magnus (M.N.C., F.G., L.S.d.V.) and Department of Medical Genetics (K.D.L.), University Medical Center Utrecht, Utrecht, the Netherlands; Divisions of Clinical and Metabolic Genetics (D.C., P.K.), Neonatology (A.G.J.), and Neuroradiology (S.B.), Department of Diagnostic Imaging The Hospital for Sick Children and Department of Paediatrics, University of Toronto, Toronto, Canada; Departments of Obstetrics and Gynecology, Laboratory Medicine, Pathobiology and Molecular Genetics (D.C.), University of Toronto, Toronto, Canada; and Division of Neonatology (E.C.), School of Clinical Sciences, St Michael's Hospital, University of Bristol, Bristol, UK.

Susan Blaser and Linda S. DeVries contributed equally to the article.

Please address correspondence to Linda S. de Vries, MD, PhD, Department Neonatology, KE 04.123.1, Lundlaan 6, 3584 EA Utrecht, the Netherlands; e-mail: l.s.devries@umcutrecht.nl

三 Indicates article with supplemental on-line table.

http://dx.doi.org/10.3174/ajnr.A5611 gen-activated protein kinase pathway, essential for cell proliferation, differentiation, and senescence. There are $>15$ genes identified so far in the RAS/mitogen-activated protein kinase signal pathway. Noonan syndrome (NS) is the most common RASopathy, and Noonan-like syndrome, Costello syndrome, cardiofaciocutaneous syndrome, and NS with multiple lentigines (formerly known as LEOPARD syndrome) are other closely related disorders..$^{2-4}$ Phenotypic features, associated risks, and severity vary widely, ranging from mild facial features to a lethal combination of congenital anomalies. ${ }^{2}$

The prenatal manifestations of RASopathies are nonspecific and include increased nuchal translucency/cystic hygroma; jugular cysts; hydrops fetalis; pleural/pericardial effusion and ascites; polyhydramnios; cardiac abnormalities, typically a dysplastic pulmonary valve; and hypertrophic cardiomyopathy. Postnatally, they present with craniofacial dysmorphism, respiratory distress, lymphatic dysplasia, cardiac defects, renal anomalies, and hypotonia. ${ }^{3,5}$ Neuroimaging features in neonates and infants with RASopathies are rare because in most cases, there is no indica- 

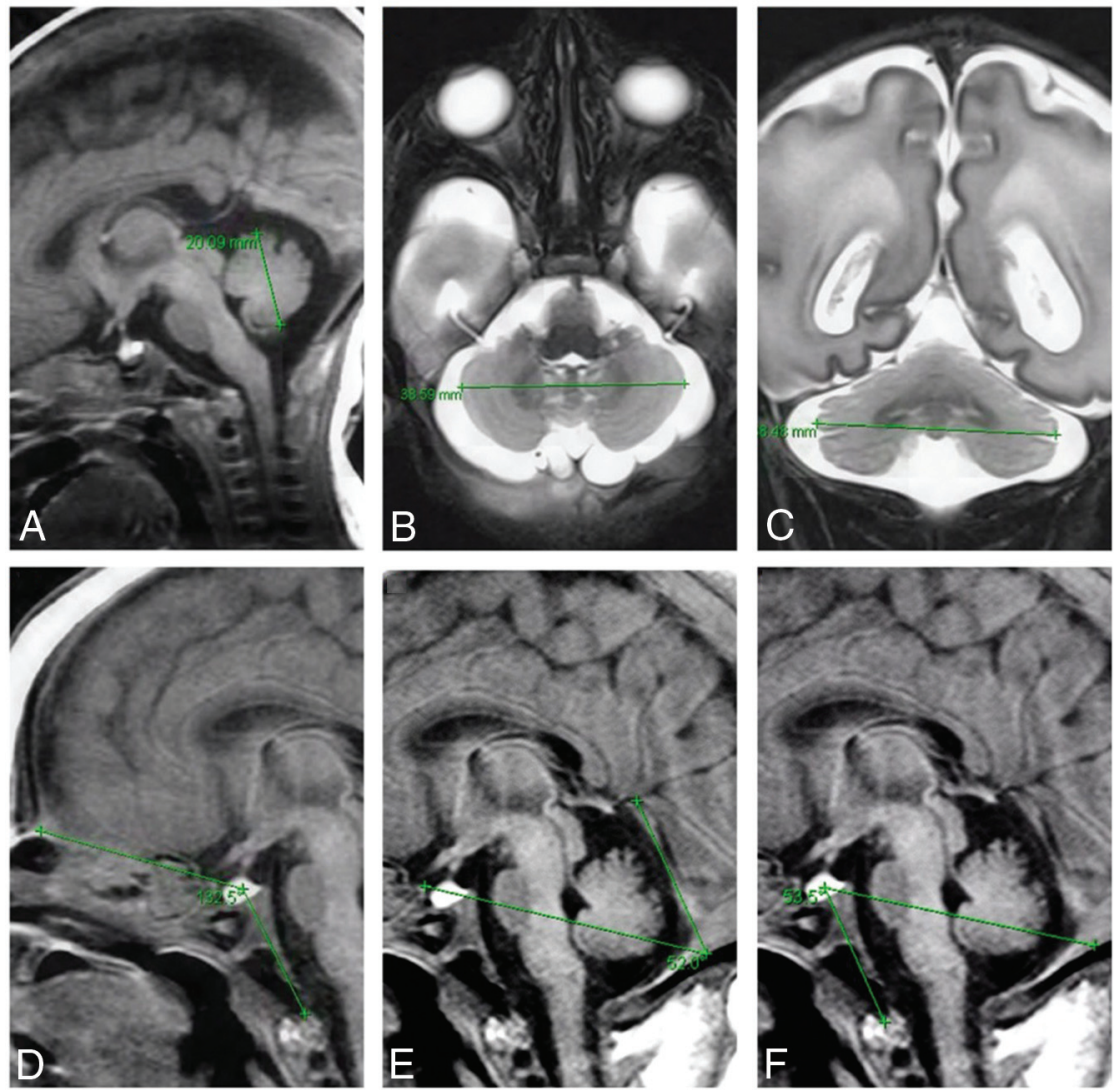

FIG 1. Measurement techniques used in the study. A, Preterm neonate, gestational age 35 weeks 6 days. Tl-weighted MR image shows measurement of the vermis height from the culmen to the uvular lobule on the midsagittal image. $B$ and $C$, Preterm neonate, gestational age 30 weeks 6 days. T2-weighted MR images show the cerebellar diameter measurement at the widest points of the cerebellum on axial and coronal images, respectively. $D$, Preterm neonate, gestational age 33 weeks 4 days. Tl-weighted image shows cranial base angle measurement with the fonticulus frontalis used as the anterior landmark; midsella, as the vertex point; and the distal tip of the ossified clivus, as the terminal point. E, Measurement of the tentorial angle between the Twining line and a line drawn through tentorium cerebelli with tuberculum sella used as the originating point. F, T1-weighted MR image shows measurement of the infratentorial angle, using a line originating from the midsella drawn through the midpoint of the dural reflection of the torcula herophili to the inner cortex, and a line drawn between the midsella and basion.

tion for brain MR imaging. Intracranial abnormalities such as Chiari malformation, syringomyelia, cerebrovascular anomalies, benign external hydrocephalus, craniosynostosis, and posterior fossa abnormalities have been reported in the pediatric and adult literature; however, to date, no observational study was conducted to define the brain abnormalities in patients with a RASopathy. ${ }^{6-9}$ We conducted a case-control study to determine the occurrence of supratentorial and posterior fossa abnormalities on brain MRIs in neonates with a RASopathy.

\section{MATERIALS AND METHODS}

An observational case-control study of neonates with a genetically confirmed RASopathy and severe clinical symptoms necessitating admission to the neonatal intensive care unit (NICU) at 3 academic centers (Wilhelmina Children's Hospital, University Medical Center Utrecht, the Netherlands; The Hospital for Sick Children, University of Toronto, Canada; and St. Michael's Hospital, University of Bristol, UK) between 2009 and 2017 was conducted. The participating centers have a long-standing practice of incor- porating brain MR imaging into the diagnostic process in severely ill neonates during their NICU course. For each neonate with a RASopathy (group 1), 2 healthy neonates with a gestational age of \pm 7 days were selected for the control group (group 2). Demographic data were extracted from the patients' files and/or hospital data base. A 1:2 casecontrol ratio was used to increase the statistical power of the analysis.

In the Wilhelmina Children's Hospital, until 2010, brain MR imaging was performed on a $1.5 \mathrm{~T}$ system (Intera or Achieva; Philips Healthcare, Best, the Netherlands), and the protocol included sagittal T1-weighted images (slice thickness, $5 \mathrm{~mm}$ ) and axial T2-weighted images (slice thickness, 2-5 mm). Between 2010 and 2017, the brain MR imaging was performed on a 3T system (Achieva; Philips Healthcare), and the recent protocol included conventional sagittal T1weighted imaging (slice thickness, 3 $\mathrm{mm}$ ), axial 3D T1-weighted imaging (slice thickness, $2 \mathrm{~mm}$ ), and axial T2weighted imaging (slice thickness, 2 $\mathrm{mm})$. The Hospital for Sick Children, Toronto, followed a similar predefined brain MR imaging protocol according to their institutional guidelines during the study period, and the MR imaging studies were all completed without pharmacologic sedation on a 1.5T Avanto scanner (Siemens, Erlangen, Germany) with 3D coronal volumetric T1-weighted and axial fast spin-echo T2-weighted images obtained at both time points. The University of Bristol followed a predefined MR imaging protocol according to their institutional guidelines, and the MR imaging study was performed on a 3T Magnetom Skyra scanner (Siemens) with 3D coronal volumetric T1-weighted and axial T2-weighted images (slice thickness, $3 \mathrm{~mm}$ ). Diffusion-weighted imaging and susceptibility-weighted imaging were performed for distinguishing ischemic and hemorrhagic lesions. DWI was acquired in the axial plane (slice thickness, $4 \mathrm{~mm}$ ), and SWI was performed using a $3 \mathrm{D}$ gradient-echo sequence with flow compensation. Only the high-quality images suitable for scoring and measurements were included in the study.

\section{MR Imaging Findings and Measurements}

MR Imaging Findings. The presence of an intraventricular and/or cerebral parenchymal hemorrhage, cerebellar hemorrhage, and punctate white matter lesions; visual assessment of the corpus callosum for hypoplasia; configuration of the splenium; gyrification of the cortex; and enlargement of the extracerebral space were evaluated by consensus reads.

AJNR Am J Neuroradiol 39:1146-52 Jun 2018 www.ajnr.org 1147 
Clinical characteristics and MRI angle measurements and assessments

\begin{tabular}{|c|c|c|c|}
\hline & $\begin{array}{l}\text { Group } 1 \\
(n=16)\end{array}$ & $\begin{array}{l}\text { Group } 2 \\
(n=32)\end{array}$ & $P$ Value ${ }^{a}$ \\
\hline Gestational age (mean) (wk) & $35.6 \pm 4.2$ & $37.3 \pm 4.5$ & $.1^{\mathrm{b}}$ \\
\hline Postmenstrual age at MRI (mean) (wk) & $37.6 \pm 4.8$ & $39.8 \pm 6.5$ & $.2^{\mathrm{b}}$ \\
\hline Vermis height (mean) (mm) & $20.5 \pm 4.4$ & $23.7 \pm 3.9$ & $.01^{\text {be }}$ \\
\hline \multicolumn{4}{|l|}{ Transcerebellar diameter (mean) } \\
\hline Axial & $48.6 \pm 9.1$ & $52.3 \pm 9.8$ & $.2^{\mathrm{b}}$ \\
\hline Coronal & $48.8 \pm 9.6$ & $52.7 \pm 9.7$ & $.2^{\mathrm{b}}$ \\
\hline Cranial base angle (mean) & $130.3^{\circ} \pm 4.0^{\circ}$ & $134.8^{\circ} \pm 5.3^{\circ}$ & $.005^{\mathrm{ce}}$ \\
\hline Tentorial angle (mean) & $55.4^{\circ} \pm 3.4^{\circ}$ & $46.6^{\circ} \pm 5.8^{\circ}$ & $<.001^{\mathrm{ce}}$ \\
\hline Infratentorial angle (mean) & $52.2^{\circ} \pm 6.5^{\circ}$ & $47.7^{\circ} \pm 4.0^{\circ}$ & $.001^{\text {be }}$ \\
\hline Intraventricular hemorrhage (No.) (\%) & $8(47)$ & $0(0)$ & $<.001^{\mathrm{de}}$ \\
\hline Cerebellar abnormality (No.) (\%) & $9(56)$ & $0(0)$ & $<.001^{\mathrm{de}}$ \\
\hline Hemorrhagic lesion & $6(67)$ & & \\
\hline Hemorrhagic-cystic lesion & $3(33)$ & & \\
\hline Enlarged extracerebral space (No.) (\%) & $13(81)$ & $0(0)$ & $<.001^{\mathrm{de}}$ \\
\hline Mild & $10(77)$ & & \\
\hline Severe & $3(23)$ & & \\
\hline Delayed cortical gyrification (No.) (\%) & $12(75)$ & $0(0)$ & $<.001^{\mathrm{de}}$ \\
\hline WMI after cerebral hemorrhage (No.) (\%) & $9(56)$ & $0(0)$ & $<.001^{\mathrm{de}}$ \\
\hline Punctate white matter lesion & $7(78)$ & & \\
\hline Cystic lesion & $2(22)$ & & \\
\hline Abnormality in DWI ( $n=12$ ) (No.) (\%) & $7(58)$ & - & \\
\hline Focal restriction & $6(86)$ & & \\
\hline Extensive restriction & $1(14)$ & & \\
\hline Corpus callosum abnormality (No.) (\%) & $4(25)$ & $0(0)$ & $<.001^{\mathrm{de}}$ \\
\hline Vertical appearance & $3(75)$ & & \\
\hline Hypoplasia & $1(25)$ & & \\
\hline
\end{tabular}

Note:-WMI indicates white matter injury.

${ }^{a}$ Adjusted for gestational age as appropriate.

${ }^{b}$ Mann-Whitney $U$ test.

c $T$ test.

${ }^{d}$ Fisher exact test.

e Significant.

MR Imaging Assessments. The vermis height was measured from the culmen to the uvular lobule on the midsagittal image, with care to avoid including the cerebellar tonsil (Fig 1A). The transverse cerebellar diameter was measured on both the coronal and axial views at the widest points of the cerebellum, traversing the dentate nuclei (Fig $1 B,-C$ ). For measurement of the cranial base angle, the fonticulus frontalis was used as the anterior landmark; midsella, as the vertex point; and the distal tip of the ossified clivus, as the terminal point (Fig 1D). The tentorial angle (TA) was measured by the angle between the Twining line and a line drawn through tentorium cerebelli. On every measurement, the tuberculum sella was used as the originating point for the TA (Fig $1 E$ ). The infratentorial angle was measured as the angle between a line originating from the midsella drawn through the midpoint of the dural reflection of torcula herophili to the inner cortex and a line drawn between the midsella and basion (Fig $1 F$ ).

Two senior neuroradiologists (M.L. and S.B.) with $>20$ years' experience in reading neonatal MRIs performed and supervised the measurements and assessed the images separately in a blinded fashion on digital PACS systems. To evaluate the interobserver agreement, we assessed 15 studies from 5 random patients and calculated the interclass correlation coefficient for each parameter. For the retrospective data analysis, the ethics committee of the University Medical Center, Utrecht, waived informed consent owing to analysis of anonymous clinical data. The same applied to

\section{RESULTS}

the control subjects from Toronto. Written parental consent was obtained in Bristol and Toronto for patients.

\section{Statistical Analysis}

Statistical analyses of the data were performed using the Statistical Package for the Social Sciences, Version 21.0 (IBM, Armonk, New York). All continuous values were presented as mean $\pm \mathrm{SD}$. Categoric values were presented as number and percentage. The Fisher exact test was used to compare categoric variables among groups. The Mann-Whitney $U$ test was used to compare nonparametric variables, and the Student $t$ test was used for the comparison of variables that showed normal distribution. The Pearson correlation coefficient was used to assess the correlation between the continuous variables. The Spearman correlation coefficient was used to search for the correlation among the non-normal variables. To evaluate the reliability of measurements across observers, we calculated the interclass correlation coefficient and classified it as good for $0.8<$ interclass correlation coefficient $<0.9$ and excellent for interclass correlation coefficient $>0.9$. Statistical significance was set at $P<.05$.

We reviewed 48 brain MR imaging studies performed at 3 academic centers between 2009 and 2017. Sixteen of these infants had a genetic diagnosis of a RASopathy (group 1), and 32 healthy neonates were enrolled as the control group (group 2). Eleven $(68.7 \%)$ neonates in group 1 were diagnosed with NS, while 5 (31.3\%) had other RASopathies. Groups were similar in terms of gestational age and postmenstrual age at the date of MR imaging (Table). Molecular genetic testing in all patients included the BRAF, HRAS, KRAS, MAP2K1, MAP2K2, PTPN11, RAF1, RIT1, SHOC2, and SOS1 genes. Eight of $16(50 \%)$ patients in group 1 had a mutation in the PTPN11 gene (NS), while there were 3 (18.8\%) with a mutation in HRAS (Costello syndrome); 1 (6.2\%), with SHOC2 (Noonan-like syndrome); 1 (6.2\%), with SOS1 (NS); 1 (6.2\%), with RAF1 (NS); 1 (6.2\%), with BRAF (cardiofaciocutaneous syndrome); and 1 (6.2\%), with RIT1 (NS). In 1 patient, the PTPN11 mutation was paternally inherited; the father was diagnosed with NS with multiple lentigines.

Data regarding white matter injury, intraventricular and cerebellar hemorrhages, and their characteristics are given in the Table. Increased rates of extracerebral space enlargement, simplification of the cortical gyrification, and white matter abnormalities were seen in group 1 (Table and Fig 2). There was a significant difference between the groups in terms of white matter lesions on conventional MR imaging, DWI, and SWI $(P<.001)$ (Table). There was $1(3.1 \%)$ neonate in group 

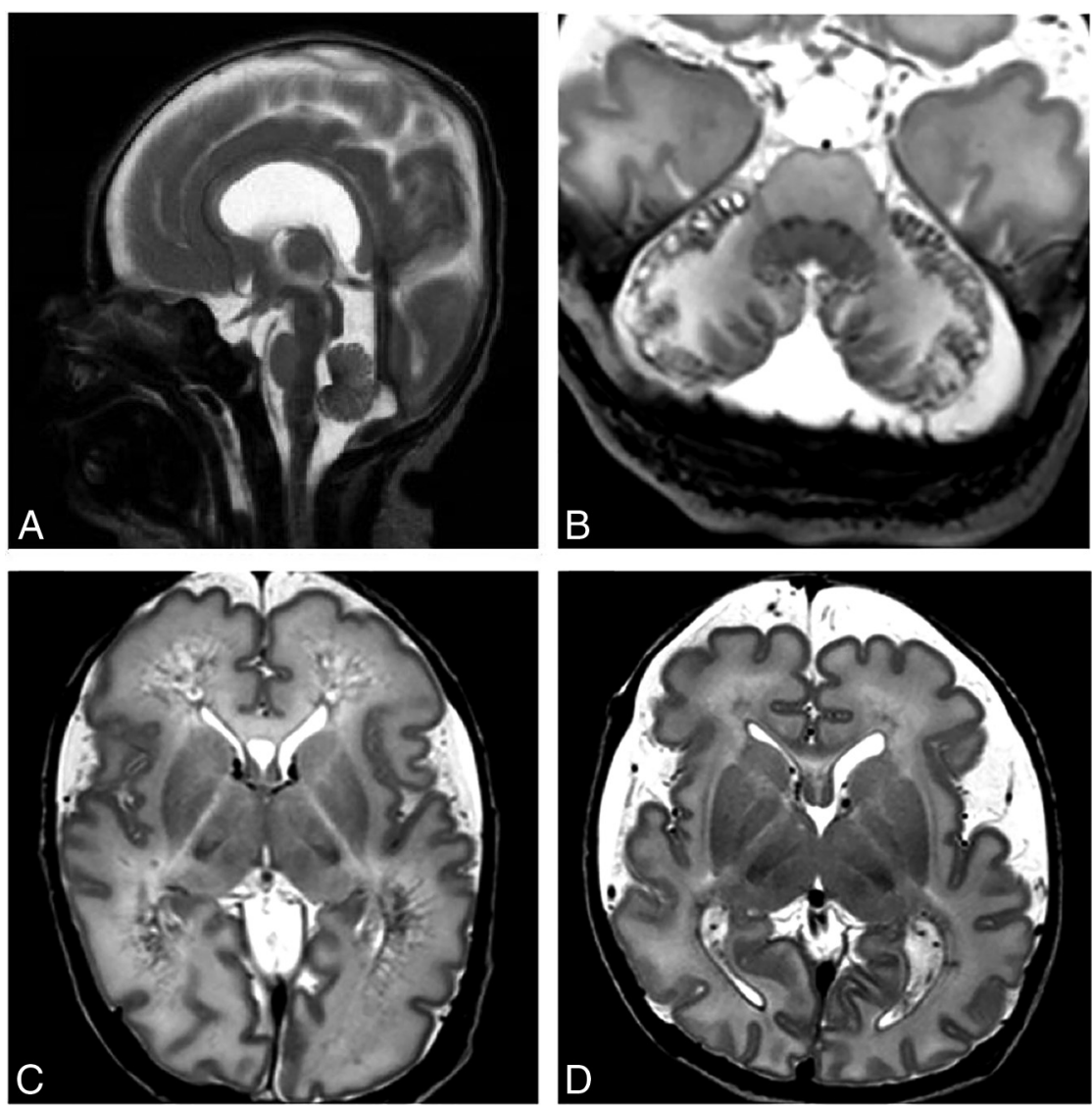

FIG 2. Characteristic MR imaging findings of neonates with a RASopathy. A, Preterm neonate, gestational age 28 weeks 6 days, MR imaging performed at postmenstrual age 30 weeks 5 days. T2-weighted midsagittal MR image shows a vertical tentorium and splenium of the corpus callosum. $B$, Preterm neonate, gestational age 34 weeks 2 days. T2-weighted axial MR image demonstrates the presence of hemorrhagic and cystic lesions in the peripheral regions of the cerebellum. C, Preterm neonate, gestational age 34 weeks 2 days. T2-weighted axial MR imaging shows a mildly enlarged extracerebral space with severe white matter injury, which evolved into extensive cysts. D, Preterm neonate, gestational age 34 weeks. Axial T2-weighted MR image shows a severely enlarged extracerebral space, punctate white matter lesions, and a small amount of blood in the lateral ventricles.

2 who was found to have a Chiari type I malformation. When the neonates in group 1 were categorized according to their mutation type [subgroup 1: PTPN11 mutation (+), subgroup 2: another type of mutation $(+)]$, there were no differences between the subgroups in terms of white matter injury, intraventricular hemorrhage, cerebellar hemorrhage, extracerebral space enlargement, or gyrification of the cerebral cortex $(P>$ .05 for all parameters).

\section{Angle Measures}

The agreement between the neuroradiologists evaluating the images showed an excellent correlation for the measurement of all angles (interclass correlation coefficient $=0.94,0.96,0.98$ for TA, cranial base angle, and infratentorial angle, respectively). When the groups were compared regarding the cerebellar measurements, the vermis height of the patients in group 1 was significantly lower than that of group $2(P=.01)$, and vermis height showed a significantly positive correlation with gestational age in both groups $(r=0.71 ; P<.001)$. Although both axial and coronal transverse cerebellar diameters of the cerebellum were reduced in group 1, they were not significantly different across the groups
$(P=.2$ for both). Both cerebellar diameters showed a positive correlation with gestational age in the study population $(r=0.73, P<.001$; and $r=0.74, P<$ .001 , respectively). Tentorial and infratentorial angles were found to be higher in group $1(P<.001$ and $P=$ .001 , respectively) (Table and Fig 3 ), and the TA showed a negative correlation with vermis height $(r=-0.30, P=.03)$. After we controlled for gestational age and categorization according to the mutation type, there were no differences between subgroup 1 and subgroup 2 regarding vermis height, TA, cranial base angle, or infratentorial angle $(P>.05$ for all parameters).

\section{Outcome}

Ten $(62.5 \%)$ patients in group 1 died during infancy due to respiratory and cardiac complications, and 7 (70\%) of these 10 infants died during their NICU stay due to multiple organ dysfunction. Only one of the surviving infants showed a normal developmental outcome. Characteristics of the patients with a RASopathy and data regarding their clinical features and outcome are given in the On-line Table.

\section{DISCUSSION}

This is the first study that describes cranial MR imaging findings in neonates with a RASopathy. We have demonstrated that these neonates had characteristic acquired and structural abnormalities in the posterior fossa, including peripheral cerebellar hemorrhage, vermis hypoplasia, and a steep tentorial configuration compared with healthy controls. Moreover, these neonates also showed an increased incidence of cerebral white matter lesions, enlarged extracerebral spaces, simplification of the cortical folding, and structural corpus callosum abnormalities involving the splenium. Apart from anecdotal pediatric and adult case reports, data on global cerebral abnormalities in neonates with a RASopathy are rare in the literature. Brasil et $\mathrm{al}^{10}$ reported cerebral abnormalities, including isolated ventriculomegaly, posterior fossa anomalies, and cerebral atrophy in infants with NS. Few studies included cerebral MR imaging, and hypoplastic corpus callosum has only been described once in a patient with RASopathy with a mutation in the NF1 gene. ${ }^{11}$ A vertical position of the splenium of the corpus callosum has also been reported in the literature. ${ }^{7}$ We have seen that the splenium of the corpus callosum showed a visually abnormal vertical configuration in 3 of our neonates and was underdeveloped in one. Furthermore, an enlarged extracerebral space, which was previously reported by Gripp et $\mathrm{al}^{7}$ in 1 patient, and delayed gyrification of the cere- 

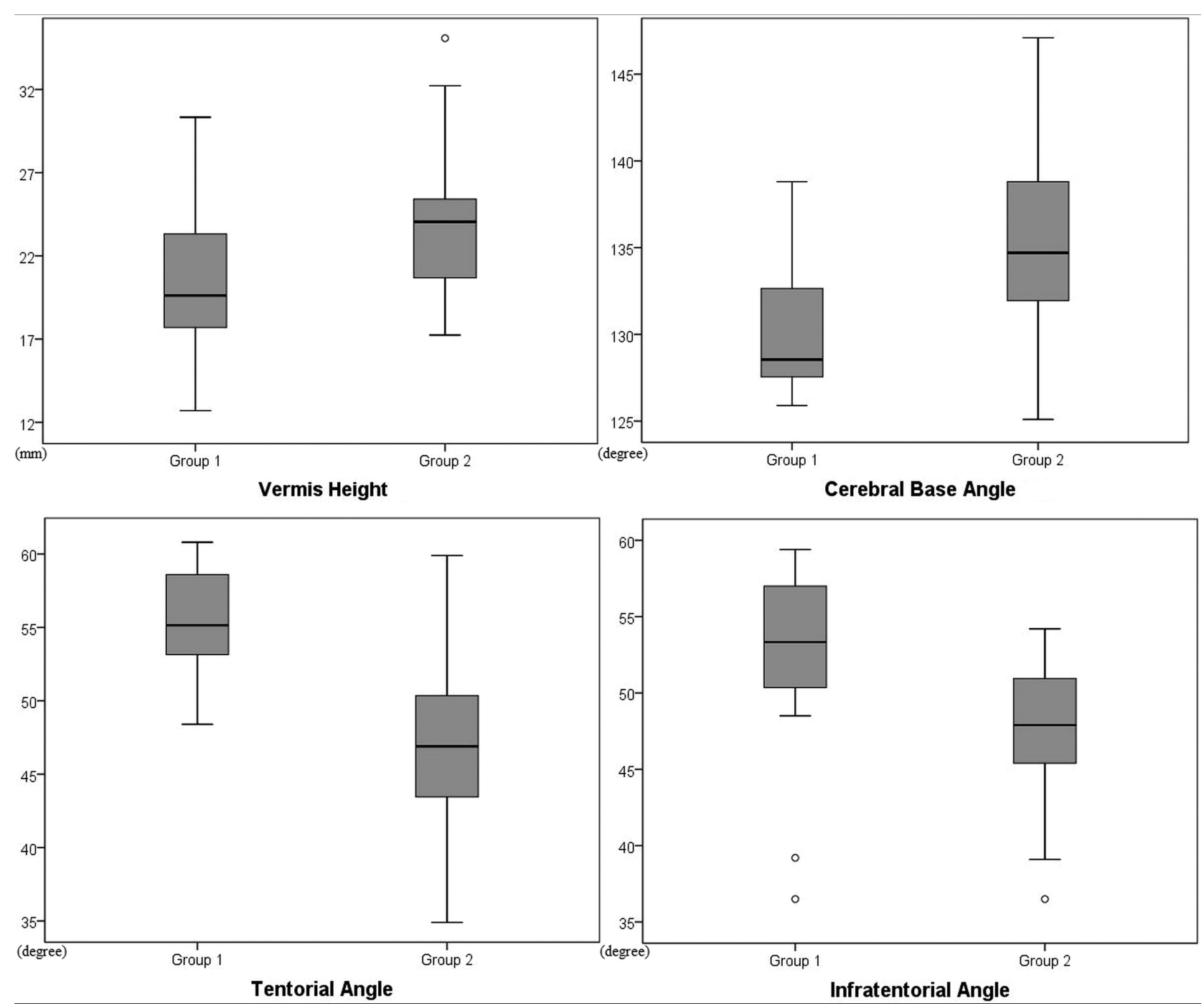

FIG 3. Boxplot graphs show the comparison of the vermis height (top left), cranial base angle (top right), tentorial angle (bottom left), and infratentorial angle (bottom right) between infants with a RASopathy and the control group.

bral cortex were present in most of our patients. To the best of our knowledge, these are all novel contributions.

Total brain tissue grows linearly prenatally, and Hüppi et $\mathrm{al}^{12}$ demonstrated an almost 3-fold increase in the global brain volume between 29 and 41 weeks postconception using volumetric MR imaging measures. The inclination of the fetal tentorium gradually increases during pregnancy, because the rate of the cerebellar growth exceeds the rate of the occipital cerebral growth. ${ }^{13}$ However, during postnatal development, the tentorium moves inferiorly while progressively decreasing the TA. ${ }^{14}$ We found that the TA of the neonates with a RASopathy was significantly higher than that of healthy controls at similar postmenstrual ages. An increased TA reflects the vertical configuration of the tentorium; however, because we have seen a negative correlation between vermis height and TA, an increased TA in these patients can be explained by the effect of a genetic disorder causing disruption of developmental processes, resulting in a permanent change in the morphology of the cranium with an effect on the growth of other structures, as defined previously. . $^{9,15,16}$

Bony structures of the skull base also undergo changes, namely retroflexion of the basicranium in the intrauterine and flexion in the postnatal period. These changes are reflected by an increase in the cranial base angle during fetal development and a subsequent decrease in the postnatal period. ${ }^{14,17}$ Any anomaly during the orderly posteroanterior ossification of the cranial structures can interfere with normal formation of the bones and soft tissues and may result in an abnormal configuration. ${ }^{18,19}$ The RAS/mitogenactivated protein kinase pathway plays a vital role in regulating components that are critical to normal development, and it is not surprising that a dysregulation in this pathway has deleterious effects on both embryonic and later stages of development, with implications on bony structures and cerebral tissues. ${ }^{1}$ It has been shown that patients with NS have various skull anomalies. ${ }^{3,20}$ Addissie et $\mathrm{al}^{21}$ reported a 2-month-old female infant with NS who developed craniosynostosis, and Ueda et $\mathrm{al}^{9}$ reported a series of 9 infants with a RASopathy and craniosynostosis. It has also been shown that infants with Costello syndrome had bone abnormalities resulting in macrocrania. ${ }^{5}$ In the present study, we have demonstrated that the cranial base angles of the infants with RASopathy were markedly depressed, a finding that reflects the abnormal skeletal development of the basicranium. Furthermore, a significantly increased infratentorial angle also 
confirms the deviant cranial cavity floor extending posteriorly, as shown in our study. Although the detailed pathophysiologic mechanism remains unknown, we speculate that rather than acquired lesions such as hemorrhages, these bone abnormalities and consequently changed angulations may result in the anomalous formation of the posterior fossa, which, in turn, changes the tentorial configuration.

In recent decades, cumulative evidence has shown that cerebellar function extends beyond sensorimotor control to relate the cerebellum with higher order functions such as attention, language, executive function, and cognition. ${ }^{22,23}$ Preterm neonates with cerebellar hemorrhage can develop cognitive impairment, and the vermis is considered especially important for cognitive outcome. ${ }^{24}$ Consistent with the literature, we have seen that the cerebellar vermis had an increase in size, as reflected by the increased vermis height, with increasing gestational age. Most interesting, we have found that neonates with a RASopathy had a small vermis compared with controls. Although the cerebellum of the infants in our study showed a trend toward a decreased diameter on coronal and axial planes, this was not statistically significant compared with healthy controls. We might speculate that the disrupting effect of these genetic conditions was more prominent on the central part of the organ, which contains more neural networks than the peripheral parts. Hemorrhages were seen in the peripheral parts of the cerebellum. It is unlikely that these hemorrhages contributed to the hypoplasia of the vermis in these infants because the hemorrhages were of recent onset and the hypoplasia was already present at birth. Further investigation is needed to find whether the hemorrhagic cerebellar lesions and the small vermis, as seen in our patients, can be used in the future as an imaging finding to better predict outcome; however, we would recommend a brain MR imaging in a neonate with a suspected diagnosis of a RASopathy, particularly when the neonatal course is complicated.

The present study has several limitations. First, due to the retrospective design of the study it is difficult to make causal inferences. Second, owing to the rarity of the severe neonatal presentation that requires NICU admission, the number of patients included in the study was relatively small. Finally, because most patients were diagnosed with NS, these results might not be generalizable for all RASopathies; however, NS is the most common type of these closely related disorders in the literature, hence, it is expected to see the same distribution in our cohort. Moreover, we have observed the same characteristic findings, consistently, in every patient in our study regardless of their final genetic diagnosis.

It is of interest that despite a wide range of neurodevelopmental outcomes in children with a RASopathy, no brain MR imaging studies have been performed previously to explore whether these could be explained by differences in brain structure and development. Our data may suggest that early MR imaging could help to better prognosticate outcome. The neonates included in our study were at the severe end of the spectrum because they required admission to a NICU. Thus, further studies are needed on those with a less complicated neonatal course. During the neonatal period, RASopathies can be challenging to diagnose because characteristic morphologic findings may not be fully established and recognition of these neuroimaging findings should facilitate the diagnosis of these conditions. ${ }^{5}$ Our study provides novel neuroimaging information that could help neonatologists, neuroradiologists, and geneticists to suspect the diagnosis and be aware of the possible complications during their follow-up. Further studies are warranted to define the exact pattern of injury and malformation in these neonates and correlate their neuroimaging findings with their neurodevelopmental outcome.

\section{CONCLUSIONS}

This study provides novel neuroimaging findings on supratentorial and posterior fossa abnormalities in neonates with a RASopathy. These structural and acquired changes in the neonatal brain might prompt the physician to consider the diagnosis of a RASopathy, and we suggest that cranial MR imaging be considered for these patients, especially when the neonatal course is complicated.

Disclosures: Peter Kannu—UNRELATED: Board Membership: Hypophosphatasia Advisory Board (Alexion). Floris Groenendaal—UNRELATED: Expert Testimony, Comments: payment for expert testimony in cases of perinatal asphyxia*; Patents (Planned, Pending or Issued): 2-iminobiotin, Comments: patent on 2-iminobiotin for neuroprotection after perinatal asphyxia*. Susan Blaser-UNRELATED: Royalties: Amirsys/Elsevier. Linda S. de Vries-UNRELATED: Employment: University Medical Center in Utrecht, Comments: I am a consult neonatologist and receive my salary from the university; Grants/Grants Pending: ZonMW (Dutch), Comments: grant for which I am co-Principal Investigator*; Payment for Lectures Including Service on Speakers Bureaus: cranial ultrasound course in London, Comments: I am a speaker at the yearly cranial ultrasound course in London. I receive an honorarium, which is not paid to me but to our neonatal neurology research foundation. The same applies to an electroencephalography meeting organized by Natus Medical this year, for which again my honorarium was paid directly to the foundation*; Royalties, Comments: I am a coauthor of 2 books for which I receive royalties: Hellström-Westas L, de Vries LS, Rosen I. An Atlas of Amplitude-Integrated EEGs in the Newborn. 2nd ed. London: Informa Health; August 2008; and Govaert P, de Vries LS. An Atlas of Neonatal Brain Sonography. 2nd ed. (CDM 182-183). London: Mac Keith Press. ISBN: 978-1-89868356-8, July 2010; Travel/Accommodations/Meeting Expenses Unrelated to activities listed, Comments: If I am an invited speaker, I usually pay for my flight and get reimbursed for these expenses. *Money paid to the institution.

\section{REFERENCES}

1. Rauen KA. The RASopathies. Annu Rev Genomics Hum Genet 2013; 14:355-69 CrossRef Medline

2. Aoki Y, Niihori T, Inoue S, et al. Recent advances in RASopathies. J Hum Genet 2016;61:33-39 CrossRef Medline

3. Roberts AE, Allanson JE, Tartaglia M, et al. Noonan syndrome. Lancet 2013;381:333-42 CrossRef Medline

4. Gelb BD, Roberts AE, Tartaglia M. Cardiomyopathies in Noonan syndrome and the other RASopathies. Prog Pediatr Cardiol 2015;39: 13-19 CrossRef Medline

5. Myers A, Bernstein JA, Brennan ML, et al. Perinatal features of the RASopathies: Noonan syndrome, cardiofaciocutaneous syndrome and Costello syndrome. Am J Med Genet A 2014;164A:2814-21 CrossRef Medline

6. Peiris A, Ball MJ. Chiari (type 1) malformation and syringomyelia in a patient with Noonan's syndrome. J Neurol Neurosurg Psychiatry 1982;45:753-54 CrossRef Medline

7. Gripp KW, Zand DJ, Demmer L, et al. Expanding the SHOC2 mutation associated phenotype of Noonan syndrome with loose anagen hair: structural brain anomalies and myelofibrosis. Am J Med Genet A 2013;161A:2420-30 CrossRef Medline

8. Hinnant CA. Noonan syndrome associated with thromboembolic brain infarcts and posterior circulation abnormalities. $\mathrm{Am} \mathrm{J} \mathrm{Med}$ Genet 1995;56:241-24 CrossRef Medline

9. Ueda K, Yaoita M, Niihori T, et al. Craniosynostosis in patients with RASopathies: accumulating clinical evidence for expanding the phenotype. Am J Med Genet 2017;173:2346-52 CrossRef Medline 
10. Brasil AS, Pereira AC, Wanderley LT, et al. PTPN11 and KRAS gene analysis in patients with Noonan and Noonan-like syndromes. Genet Test Mol Biomarkers 2010;14:425-32 CrossRef Medline

11. Nyström AM, Ekvall S, Stromberg B, et al. A severe form of Noonan syndrome and autosomal dominant cafe-au-lait spots: evidence for different genetic origins. Acta Paediatr 2009;98:693-98 CrossRef Medline

12. Hüppi PS, Warfield S, Kikinis R, et al. Quantitative magnetic resonance imaging of brain development in premature and mature newborns. Ann Neurol 1998;43:224-35 CrossRef Medline

13. Moss ML, Noback CR. Differential growth of the human brain. J Comp Neurol 1956;105:539-51 CrossRef Medline

14. Rehder R, Yang E, Cohen AR. Variation of the slope of the tentorium during childhood. Childs Nerv Syst 2016;32:441-50 CrossRef Medline

15. Meyer-Marcotty P, Böhm H, Linz C, et al. Three-dimensional analysis of cranial growth from 6 to 12 months of age. Eur J Orthod 2014;36:489-96 CrossRef Medline

16. Cao H, Alrejaye $\mathrm{N}$, Klein OD, et al. A review of craniofacial and dental findings of the RASopathies. Orthod Craniofac Res 2017;20: 32-38 CrossRef Medline

17. Jeffery N, Spoor F. Brain size and the human cranial base: a prenatal perspective. Am J Phys Anthropol 2002;118:324-40 CrossRef Medline
18. Nemzek WR, Brodie HA, Hecht ST, et al. MR, CT, and plain film imaging of the developing skull base in fetal specimens. AJNR Am J Neuroradiol 2000;21:1699-706 Medline

19. Matras H, Watzek G, Perneczky A. Cephalometric observations in premature craniosynostosis. J Maxillofac Surg 1977;5:298-303 CrossRef Medline

20. Naficy S, Shepard NT, Telian SA. Multiple temporal bone anomalies associated with Noonan syndrome. Otolaryngol Head Neck Surg 1997;116:265-67 CrossRef Medline

21. Addissie YA, Kotecha U, Hart RA, et al. Craniosynostosis and Noonan syndrome with KRAS mutations: expanding the phenotype with a case report and review of the literature. Am J Med Genet A 2015;167A:2657-63 CrossRef Medline

22. Sokolov AA, Miall RC, Ivry RB. The cerebellum: adaptive prediction for movement and cognition. Trends Cogn Sci 2017;21:313-32 CrossRef Medline

23. Limperopoulos $\mathrm{C}$. The vulnerable immature cerebellum. Semin Fetal Neonatal Med 2016;21:293-94 CrossRef Medline

24. Stoodley CJ, Limperopoulos C. Structure-function relationships in the developing cerebellum: evidence from early-life cerebellar injury and neurodevelopmental disorders. Semin Fetal Neonatal Med 2016;21:356-64 CrossRef Medline 